\title{
TaClpS1, negatively regulates wheat resistance against Puccinia striiformis f. sp. tritici
}

\author{
Qian Yang ${ }^{\dagger}$, Md Ashraful Islam ${ }^{\dagger}$ Kunyan Cai, Shuxin Tian, Yan Liu, Zhensheng Kang ${ }^{*}$ and Jun Guo* (D)
}

\begin{abstract}
Background: The degradation of intracellular proteins plays an essential role in plant responses to stressful environments. ClpS1 and E3 ubiquitin ligase function as adaptors for selecting target substrates in caseinolytic peptidase (Clp) proteases pathways and the 265 proteasome system, respectively. Currently, the role of E3 ubiquitin ligase in the plant immune response to pathogens is well defined. However, the role of ClpS1 in the plant immune response to pathogens remains unknown.

Results: Here, wheat (Triticum aestivum) ClpS1 (TaClpS1) was studied and resulted to encode 161 amino acids, containing a conserved ClpS domain and a chloroplast transit peptide (1-32 aa). TaClpS1 was found to be specifically localized in the chloroplast when expressed transiently in wheat protoplasts. The transcript level of TaClpS1 in wheat was significantly induced during infection by Puccinia striiformis f. sp. tritici (Pst). Knockdown of TaClpS1 via virus-induced gene silencing (VIGS) resulted in an increase in wheat resistance against Pst, accompanied by an increase in the hypersensitive response (HR), accumulation of reactive oxygen species (ROS) and expression of TAPR1 and TaPR2, and a reduction in the number of haustoria, length of infection hypha and infection area of Pst. Furthermore, heterologous expression of TaClpS1 in Nicotiana benthamiana enhanced the infection by Phytophthora parasitica.
\end{abstract}

Conclusions: These results suggest that TaClpS1 negatively regulates the resistance of wheat to Pst.

Keywords: TaClpS1, Puccinia striiformis f. sp. tritici, Wheat, Virus-induced gene silencing, Heterologous expression

\section{Background}

To ensure their survival in nature, plants must evoke many complicated mechanisms to cope with biotic and abiotic stresses. An increasing number of studies reveal the essential role that the degradation of intracellular proteins plays in plant responses to stressful environments. The protein degradation pathways include ubiquitin-26S proteasome system (UPS) and caseinolytic peptidase (Clp) proteases $[1,2]$. The two protein

\footnotetext{
*Correspondence: kangzs@nwsuaf.edu.cn; quojunwgq@nwsuaf.edu.cn ${ }^{+}$Qian Yang and Md Ashraful Islam contributed equally to this work. State Key Laboratory of Crop Stress Biology for Arid Areas, College of Plant Protection, Northwest A\&F University, Yangling 712100, Shaanxi, P. R. China
}

degradation machineries both consist of large multisubunit proteolytic complexes.

The first process in degradation of proteins by UPS is ATP-dependent ubiquitination, which involves the action of at least three main enzymes for selecting target substrates [3]: ubiquitin-activating enzymes (E1), ubiquitin-conjugating enzymes (E2), and ubiquitin ligases (E3). Studies reveal that ubiquitination during UPS is implicated in many biological processes in plants, including hormone signaling, growth and development, circadian rhythm control and cell cycle [4, 5]. Recent studies have indicated that ubiquitination and E3 ubiquitin ligases are involved in plant immunity to pathogens. For instance, a RING finger E3 ubiquitin ligase, BLAST

(c) The Author(s). 2020 Open Access This article is licensed under a Creative Commons Attribution 4.0 International License, which permits use, sharing, adaptation, distribution and reproduction in any medium or format, as long as you give appropriate credit to the original author(s) and the source, provide a link to the Creative Commons licence, and indicate if changes were made. The images or other third party material in this article are included in the article's Creative Commons licence, unless indicated otherwise in a credit line to the material. If material is not included in the article's Creative Commons licence and your intended use is not permitted by statutory regulation or exceeds the permitted use, you will need to obtain permission directly from the copyright holder. To view a copy of this licence, visit http://creativecommons.org/licenses/by/4.0/ The Creative Commons Public Domain Dedication waiver (http://creativecommons.org/publicdomain/zero/1.0/) applies to the data made available in this article, unless otherwise stated in a credit line to the data. 
AND BTH-INDUCED1 (OsBBI1), was found to positively regulate resistance against Magnaporthe oryzae by modifying the rice cell wall [6]; SPL1, a rice U-box protein with E3 ubiquitin ligase activity, negatively regulates cell death and innate immunity against $M$. oryzae and Xanthomonas oryzae pv. oryzae (Xoo) [7]; SGT1, an ubiquitin ligase-associated protein, is required for induction of important defense mechanisms, including $\mathrm{R}$ genemediated defense mechanisms, systemic acquired resistance and basal defense [8]. These studies hinted that factors, which play an important role in selecting target proteins in protein degradation machineries, participate in regulating plant resistance to pathogens. The caseinolytic peptidase (Clp) protease-mediated protein degradation system initially discovered in bacteria consists of a proteolytic protein (mainly $\mathrm{ClpP}$ ) and some regulatory AAA+ (ATPase associated with diverse cellular activities) proteins [9]. Importantly, regulatory AAA+ proteins use adaptor proteins to recognize and target specific substrates for degradation. For example, in Escherichia coli, the regulatory AAA+ protein ClpA uses the adaptor $\mathrm{ClpS}$ to recognize and target substrates for degradation by ClpAP protease [10-12]. These studies in $E$. coli indicated that the adaptor ClpS plays a central role in selecting target substrates for degradation by the ClpAP protease, which is the same as the function of E3 ubiquitin ligase in UPS. ClpS from E. coli was found to contain two conserved regions by analyzing the crystal structure of ClpS, and they were shown to be involved in the interaction with ClpA and substrates, respectively [13, 14]. Intriguingly, the region involved in the interaction with substrates shared secondary structure homology with E3 ubiquitin ligases in UPS [15]. Moreover, previous phylogenetic analyses of $\mathrm{ClpS}$ proteins revealed evolutionary linkages among bacteria, cyanobacteria and plants [15, 16]. Those studies raise the question: could ClpS proteins in plants participate in regulating plant resistance to pathogens just as E3 ubiquitin ligases function in plants?

The plant chloroplast Clp system comprises a heterooligomeric protease core complex consisting of five proteolytic subunits (ClpP1 and ClpP3-6) and four different subunits (ClpR1-4), ATP-dependent chaperones ClpC1/ 2 and $\mathrm{ClpD}$, and an adaptor protein ClpS1, a redefinition of plant ClpS proteins based on subsequent phylogenetic analyses [17, 18]. In Arabidopsis, several direct candidate chloroplast AtClpS1 substrates have been identified based on affinity purification methods, including glutamyl-tRNA reductase (GLUTR) and four enzymes in the shikimate pathway [17, 19]. Moreover, the interaction of ClpS1 with the candidate substrates was strictly dependent on two conserved $\mathrm{ClpS1}$ residues involved in recognizing and binding substrates, indicating that ClpS1 is a conserved substrate selector for the chloroplast Clp protease system [17]. In addition, AtClpS1 was reported to also interact with chloroplast chaperones $\mathrm{ClpC} 1,2$ and adaptor $\mathrm{ClpF}$, suggesting a model in which ClpS1 and $\mathrm{ClpF}$ form a binary adaptor for selective substrate recognition and delivery to $\mathrm{ClpC}[17,18]$. However, these reports do not reveal that the plant ClpS1 protein participates in regulating plant resistance to pathogens.

Wheat stripe rust, caused by Puccinia striiformis $\mathrm{f}$. sp. tritici (Pst), is one of the most widespread and destructive diseases of wheat worldwide [20]. In this study, the function of $\mathrm{ClpS} 1$ in plant responses to pathogens, based mainly on the interaction system of wheat and the stripe rust pathogen was studied. A ClpS1 gene from Triticum aestivum cv. Suwon 11, designated TaClpS1 was studied. The transcript level of TaClpS1 in wheat was induced by the Pst isolate CYR23 [21]. Knocking down TaClpS1 expression in wheat by virus-induced gene silencing (VIGS) attenuated Pst infection intensity and enhanced the accumulation of reactive oxygen species (ROS). Moreover, transient expression of TaClpS1 in $N$. benthamiana facilitated the infection of Phytophthora parasitica. These results suggested that TaClpS1 most likely serves as an enhancer of disease in plant which ultimately increases plant susceptibility to pathogen.

\section{Results}

\section{Identification of the wheat TaClpS1}

In this study, wheat TaClpS1 was identified using the protein sequence of Arabidopsis AtClpS1 (GenBank accession no. NP_564937.1) blasting the hexaploid wheat genome databases (http://plants.ensembl.org/index.html). The results showed that six homologous sequences of AtClpS1 in wheat were located on chromosomes $2 \mathrm{~A}, 2 \mathrm{~B}, 2 \mathrm{D}, 3 \mathrm{~A}$, $3 \mathrm{~B}$ and 3D, respectively. Phylogenetic analysis of the ClpS1 proteins from various plant species showed that the TaClpS1-2A, TaClpS1-2B, and TaClpS1-2D proteins are closely related to $\mathrm{ClpS1}$ proteins from other plants, including Arabidopsis, Zea mays and Oryza sativa. The TaClpS1H-3A, TaClpS1H-3B, and TaClpS1H-3D proteins are not included in this group (Fig. 1a). Therefore, in this study we focused on the function of TaClpS1-2A, TaClpS1-2B, and TaClpS1-2D.

The predicted proteins TaClpS1-2A, TaClpS1-2B, and TaClpS1-2D all encoded 161 amino acids, with a sequence identity of $98.77 \%$. Sequences of the predicted proteins TaClpS1-2A, TaClpS1-2B, and TaClpS1-2D were determined to contain a conserved $\mathrm{ClpS}$ domain by using Pfam online (http://pfam.xfam.org/) (Fig. 1b). Based on the above analyses, it was concluded that the identified genes TaClpS1-2A, TaClpS1-2B, and TaClpS1-2D encode the ClpS1 protein in wheat.

\section{TaClpS1 is localized in the chloroplast of wheat}

To determine the subcellular location of TaClpS1, using localizer online (http://localizer.csiro.au/), 


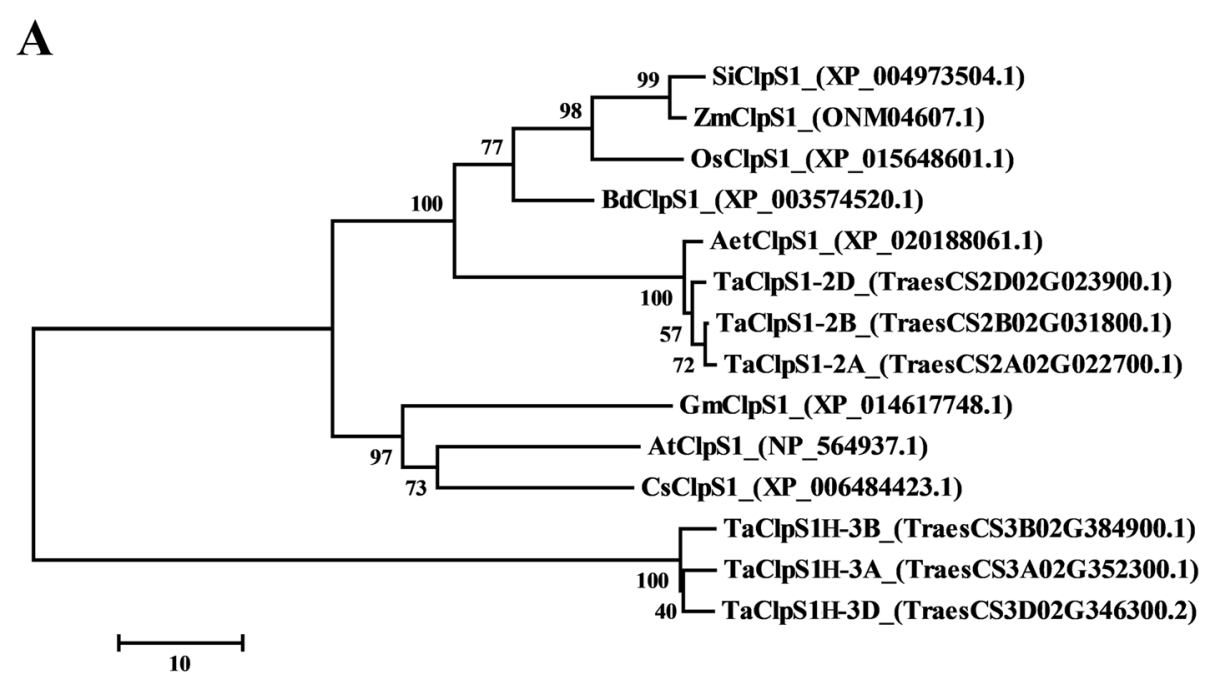

B

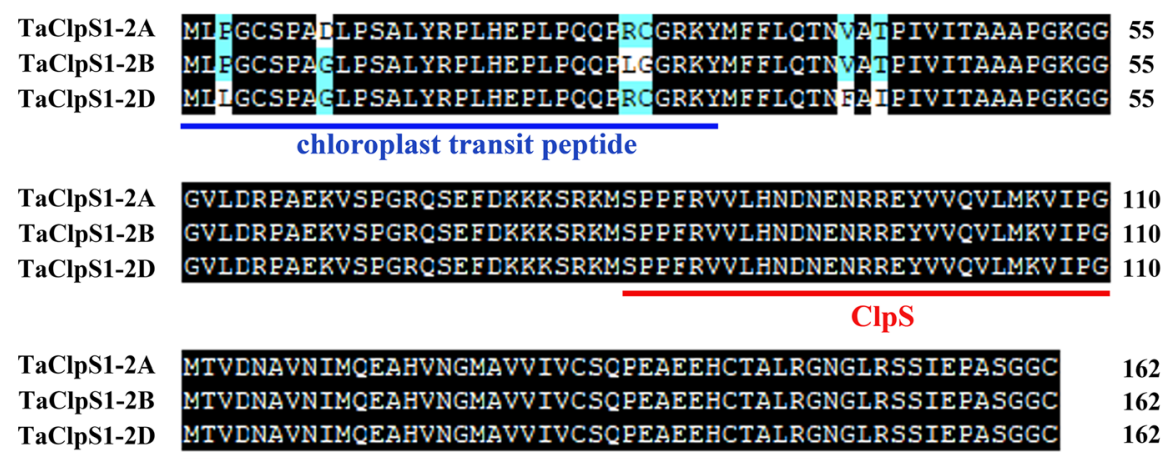

Fig. 1 Phylogenetic analysis and protein sequence alignment of TaClpS1. a Phylogenetic analysis of ClpS1 proteins from representative species. The phylogenetic tree was developed based on the maximum-likelihood method using MEGA5 software. Ta, Triticum aestivum; At, Arabidopsis thaliana; Bd, Brachypodium distachyon; Os, Oryza sativa; Zm, Zea mays; Si, Setaria italica; Gm, Glycine max; Cs, Citrus sinensis; Aet, Aegilops tauschii. Protein sequences of TaClpS1 from chromosome 2A, 2B, and 2D of wheat were denoted as TaClpS1-2A, TaClpS1-2B, and TaClpS1-2D, respectively. Sequences from chromosome 3A, 3B, 3D of wheat were denoted as TaClpS1H-3A, TaClpS1H-3B, and TaClpS1H-3D, respectively. b Protein sequence alignment of TaClpS1-2A, TaClpS1-2B and TaClpS1-2D. Light blue or colorless shade denotes the amino acid dissimilarities within TaClpS1-2A, TaClpS1-2B and TaClpS1-2D. Regions labelled by red and blue lines represent the ClpS1 domain and the chloroplast transit peptide, respectively

TaClpS1-2A, TaClpS1-2B, and TaClpS1-2D were predicted to contain a chloroplast transit peptide (1-32 aa) (Fig. 1b). Considering that TaClpS1-2A, TaClpS1-2B, and TaClpS1-2D are highly conserved in amino acid sequence, TaClpS1-2A was selected as a representative of all $\mathrm{TaClpS} 1$ and generated the fusion constructs pCAMBIA1302: TaClpS1-GFP. TaClpS1-2A lacking the chloroplast transit peptide (1-32 aa) (TaClpS1 $\Delta$ ) was fused into vector pCAMBIA1302 to generate pCAMBIA1302: $\mathrm{TaClpS1} 1-$ GFP, which was used as a negative control. These constructs were transformed into $N$. benthamiana leaves via $A$. tumefaciens infiltration. Confocal microscopy showed that TaClpS1-GFP was localized in the nucleus, cytomembrane and chloroplast of $N$. benthamiana, while control pCAMBIA1302: GFP and pCAMBIA1302: TaClpS1 $\triangle$-GFP were localized in the nucleus, cytomembrane and cytoplasm (Fig. 2a).

To further confirm the localization of TaClpS1 in wheat cells, the fusion constructs pCaMV35S: TaClpS1GFP and pCaMV35S: TaClpS1 $1 \Delta$-GFP were generated and transformed into wheat protoplasts by polyethyleneglycol (PEG)-calcium. GFP fluorescence signals of pCaMV35S: TaClpS1 1 -GFP and pCaMV35S: GFP appeared in the nucleus, cytomembrane and cytoplasm of wheat protoplasts. In contrast to the results observed in $N$. benthamiana leaves, GFP fluorescence signals of TaClpS1-GFP aggregated mainly in chloroplasts of wheat protoplasts (Fig. 2b). 


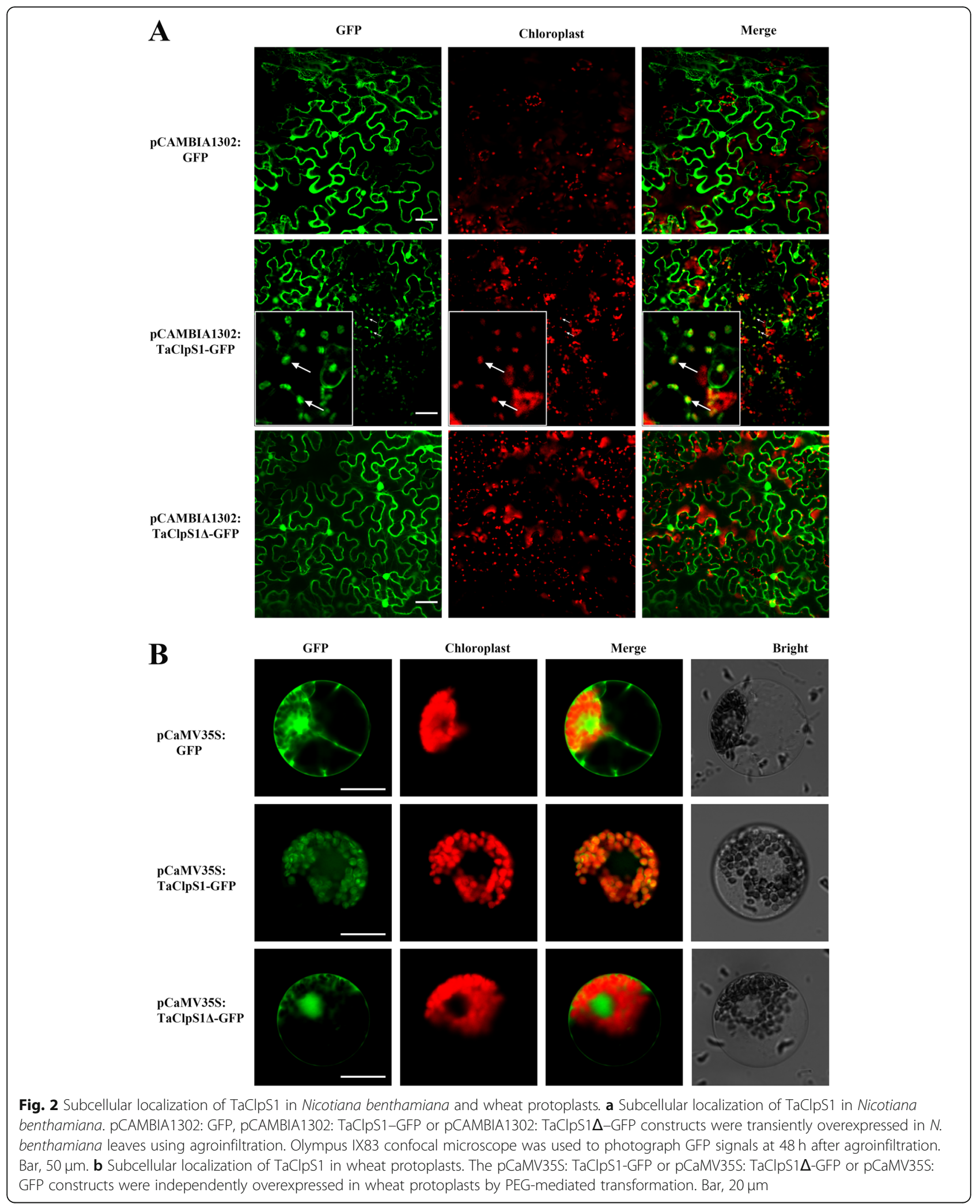




\section{Relative transcript levels of $\mathrm{TaClpS1}$ at different stages} during Pst infection

To explore the role of TaClpS1 during Pst infection, the qRT-PCR assay was performed to examine the relative transcript levels of TaClpS1 at different stages during infection by Pst isolate CYR23. qRT-PCR data showed that the transcript levels of TaClpS1 increased as early as 12 $\mathrm{h}$ post-inoculation (hpi), continued to increase to $24 \mathrm{hpi}$, and subsequently diminished at $48 \mathrm{hpi}$, before the transcript levels rose again at 96 and 120 hpi (Fig. 3). The qRT-PCR results clearly indicate that the transcript levels of TaClpS1 in wheat were induced during Pst infection, suggesting that TaClpS1 participates in the interaction between wheat and Pst.

TaClpS1 is a negative regulator of wheat resistance to Pst To examine whether TaClpS1 is involved in regulating the wheat defense resistance against Pst, the Barley Stripe Mosaic Virus (BSMV)-induced gene silencing (VIGS) strategy was used. Two specific fragments (TaClpS1-1/2as) were designed to specifically silence all three copies of the endogenous TaClpS1 gene (TaClpS1-2A/2B/2D) in Su11 wheat (Additional file 1: Fig. S1). All the wheat leaves inoculated with BSMV: $\gamma$ (negative control) or BSMV: TaClpS1-1/2as displayed mild chlorotic mosaic symptoms at 12 dpi (Fig. 4a). Subsequently, the fourth leaves of silenced wheat were inoculated with incompatible Pst isolate CYR23. The leaves

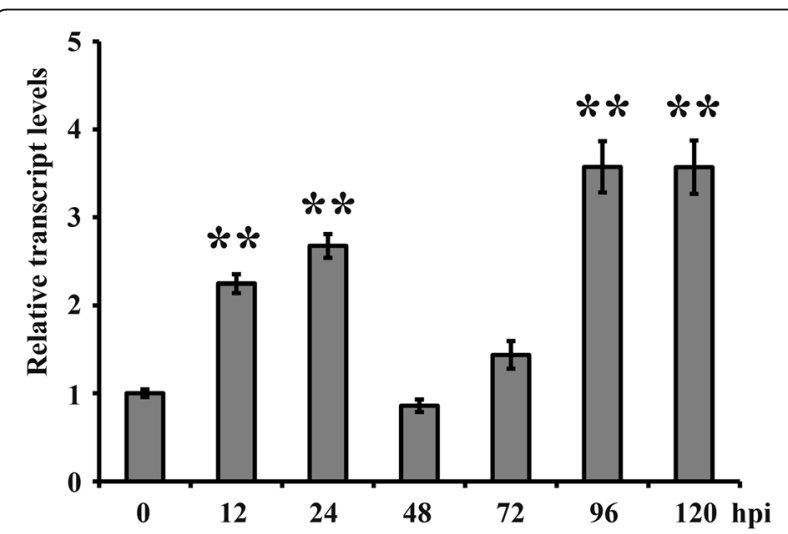

Fig. 3 Analysis of TaClpS1 expression in wheat leaves inoculated with Pst CYR23. Wheat leaves (Su11) inoculated with Pst CYR23 were sampled at various times, representing the different stages of Pst infection. Mock was sampled at 0 hpi. The comparative threshold $\left(2^{-\Delta \Delta C \mathrm{~T}}\right)$ approach was used to analyze the relative transcript levels of TaCIPS1. Data were normalized with the transcript level of wheat elongation factor, TaEF-1a, and visualized as the fold changes compared to control at $0 \mathrm{hpi}$. Each data represent the means \pm standard errors of three independent biological replicates. Double asterisks denote the significant difference $(P<0.01)$ from 0 hpi calculated by unpaired two-tailed Student's $t$-test. Hpi, hours post inoculation inoculated with CYR23 displayed hypersensitive response (HR) symptoms in negative controls and BSMV: TaClpS1-1/2as silenced lines (Fig. 4a). qRTPCR confirmed that the transcript levels of TaClpS1 were significantly reduced in BSMV: TaClpS1-1/2as silenced lines compared with BSMV: $\gamma$ treated wheat at 0,24 and 120 hpi (Fig. 4b). Pathogenesis-related $(P R)$ genes, including $P R 1$ and $P R 2$, are generally considered as marker genes in HR and are necessary for resistance of plants to pathogens [21, 22]. Then, the transcript levels of TaPR1 and TaPR2 were analyzed in TaClpS1-1/2as silenced lines and BSMV: $\gamma$ treated wheat inoculated with CYR23 at 0,24 and 120 hpi. Our qRT-PCR results showed that the transcript levels of TaPR1 (Fig. 4c) and TaPR2 (Fig. 4d) were notably increased in TaClpS1-1/2as silenced lines compared with that in BSMV: $\gamma$ treated wheat. In addition, the areas of necroses and $\mathrm{H}_{2} \mathrm{O}_{2}$ accumulation induced by inoculation with CYR23 in wheat leaves were measured at $24 \mathrm{hpi}$. As shown in Fig. 5, $\mathrm{H}_{2} \mathrm{O}_{2}$ accumulation per infection site (Fig. 5a, b) and the necrotic area (Fig. 5c, d) in TaClpS1-1/2as silenced wheat were obviously greater than that in BSMV: $\gamma$ treated wheat. Taken together, these results revealed that TaClpS1 stimulates the Pst infection in wheat, which finally increased plant susceptibility during wheat-Pst incompatible interaction.

\section{Silencing TaClpS1 significantly inhibits the growth of Pst}

In addition to analyze necroses and $\mathrm{H}_{2} \mathrm{O}_{2}$ accumulation, histological analysis of mycelial structures of Pst was performed in wheat leaves infected with CYR23 at 24 and 120 hpi (Fig. 6a). The numbers of haustoria (Fig. 6b), hyphal lengths (Fig. 6c) and hyphal infection area (Fig. 6d), which are indicators to assess fungal expansion ability, were strictly reduced in TaClpS1-1/2as silenced wheat compared with that in BSMV: $\gamma$ treated wheat. These results indicated that silencing TaClpS1 diminished the growth of Pst.

\section{TaClpS1 negatively regulates disease resistance of $N$. benthamiana to Phytophthora parasitica}

To further verify the conclusion that TaClpS1 negatively regulates plant resistance against pathogens, firstly the interaction of the model plant $N$. benthamiana and oomycete Phytophthora parasitica was examined. In this experiment, $A$. tumefaciens carrying plasmid pCAMBIA1302: TaClpS1-GFP or pCAMBIA1302: GFP (negative control) were infiltrated, and then $P$. parasitica mycelial plugs were placed onto the infiltrated $N$. benthamiana leaves. As expected, compared with the pCAMBIA1302: GFP negative control, lesion diameters of leaves expressing pCAMBIA1302: TaClpS1-GFP were 


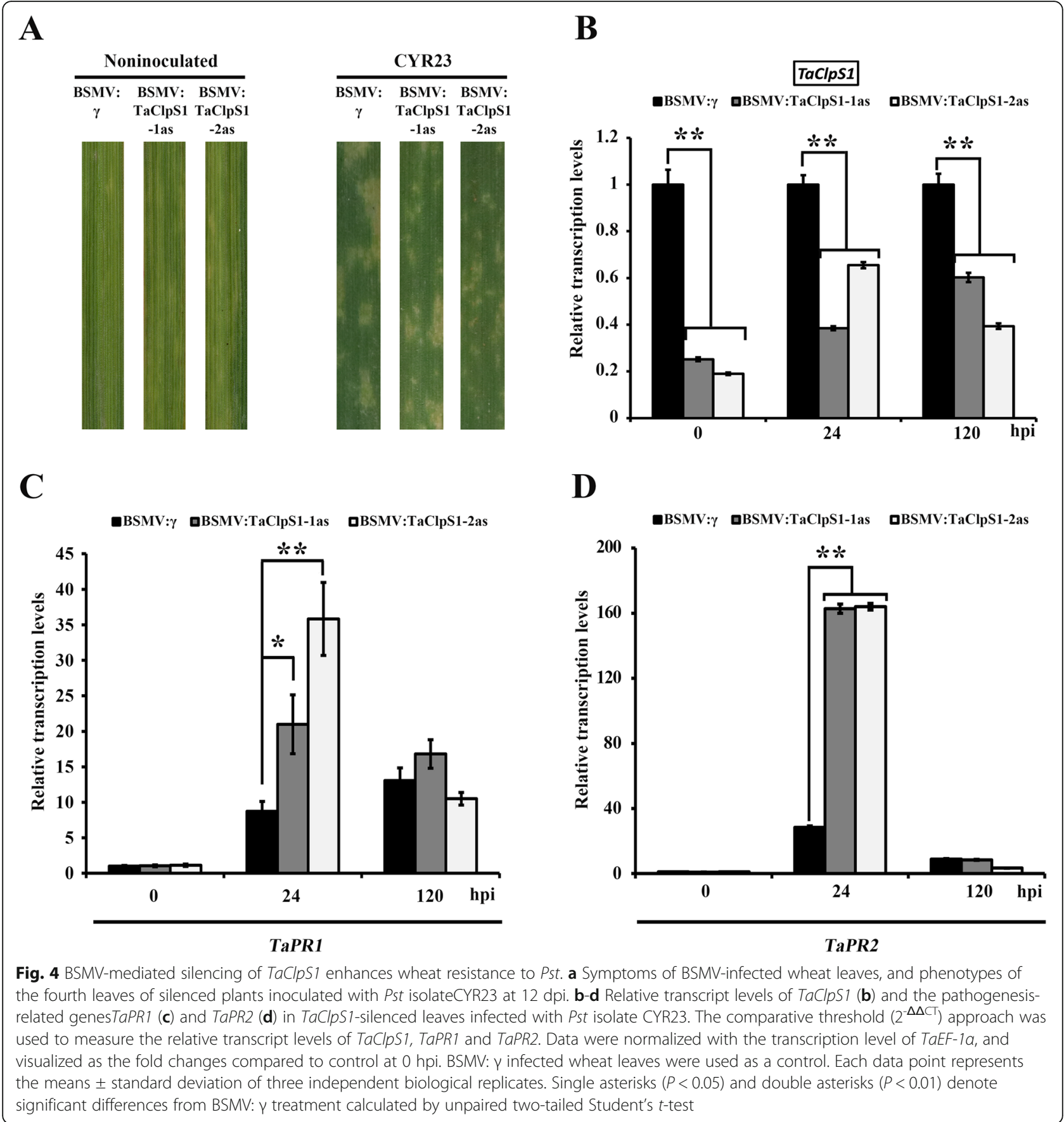

significantly larger, demonstrating that ectopic expression of TaClpS1 in $N$. benthamiana can enhance $P$. parasitica infection (Fig. 7a, b). In addition, VIGS strategy was utilized to silence TaClpS1 and then inoculated with Pst virulent isolate CYR31. As shown in Fig. 7c, the uredia on TaClpS1 silenced leaves inoculated with CYR31 at $12 \mathrm{hpi}$ were less than that on negative controls. Moreover, qRTPCR confirmed that the transcript levels of TaClpS1 were significantly reduced in BSMV: TaClpS1-1/2as silenced lines compared with BSMV: $\gamma$ treated wheat at 24 and 120 hpi (Fig. 7d). Taken together, these results indicate that TaClpS1 negatively regulates disease resistance of plants.

\section{TaClpS1 interacts with TaHEMA1 by yeast two-hybrid} assay

In Arabidopsis, GluTR, encoded by gene HEMA1 (AT1G58290), was identified as a candidate substrate of AtClpS1 [17]. To confirm whether glutamyl-tRNA reductase is a substrate of $\mathrm{TaClpS1}$ in wheat, yeast two-hybrid $(\mathrm{Y} 2 \mathrm{H})$ technique was used. Firstly, a 


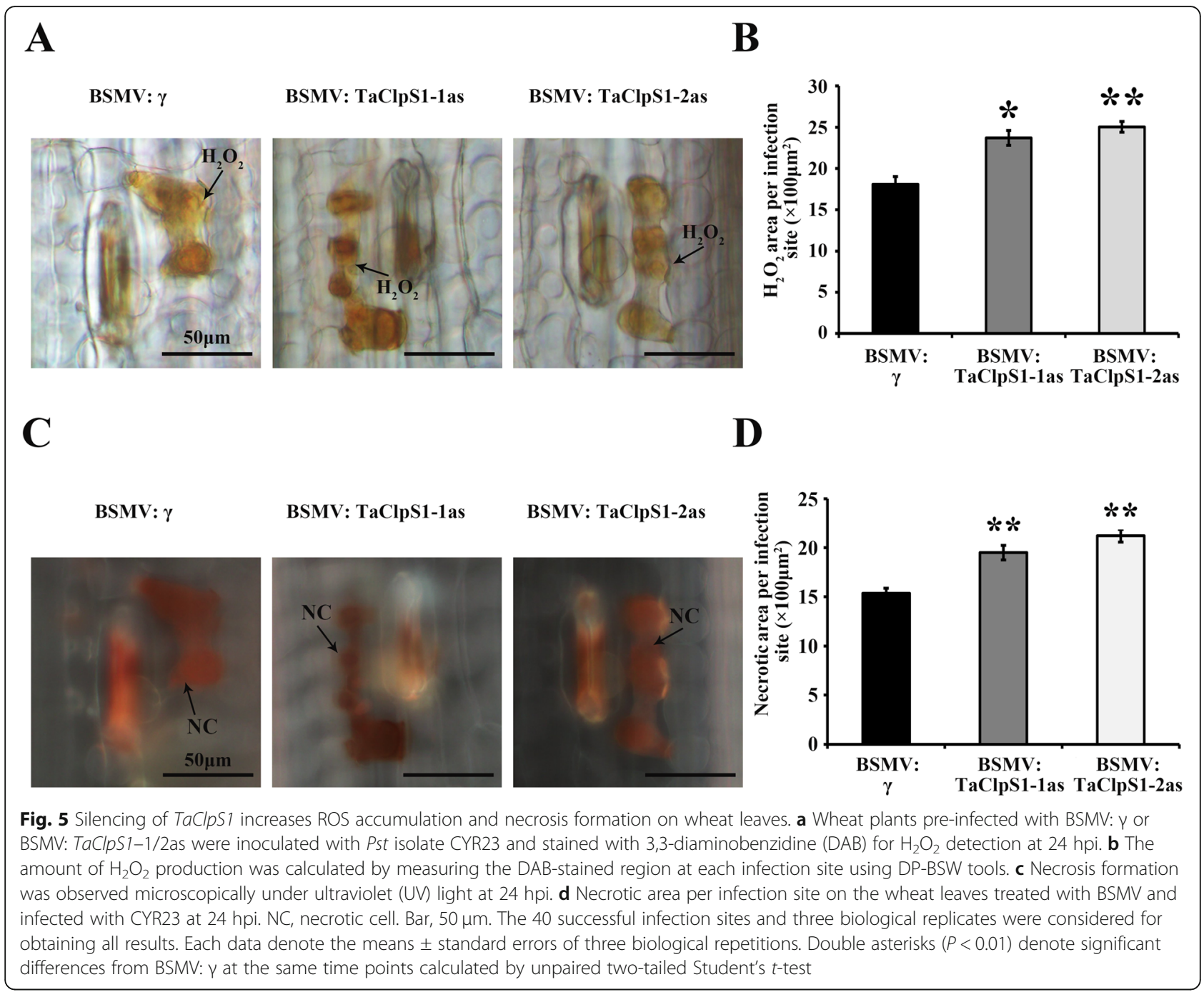

BLAST search using the protein sequence of Arabidopsis HEMA1 as a query showed that the hexaploid wheat genome contains three homologous sequences of AtHEMA1, which were located on chromosomes $1 \mathrm{~A}, 1 \mathrm{~B}$ and $1 \mathrm{D}$, respectively. Subsequently, the three homologous genes were named TaHEMA1 based on phylogenetic tree constructed with HEMA1 proteins from various plant species (Additional file 2: Fig. S2A). Considering that the three copies of TaHEMA1 are highly conserved in amino acid sequence with 98.37\% identity (Additional file 2: Fig. S2B), TaHEMA1 in chromosomes $1 \mathrm{~B}$ was selected as a representative to perform $\mathrm{Y} 2 \mathrm{H}$ assay. In $\mathrm{Y} 2 \mathrm{H}$ assay, only yeast cell carrying TaHEMA1 and TaClpS1 could grow normally on SDLeu-Trp-His-Ade medium containing $\mathrm{X}$ - $\alpha$-gal and appeared blue (Additional file 2: Fig. S2C), indicating that TaClpS1 interacts with TaHEMA1. Overall, these results suggest GluTR encoded by TaHEMA1 could be a candidate substrate of $\mathrm{TaClpS} 1$ in wheat.

\section{Discussion}

In this study, the TaClpS1 was isolated from T. aestivum Suwon11 leaves. TaClpS1 is homologous with ClpS1 proteins from various plants, indicating a high sequence conservation of ClpS1 among different plant species. The numbers of uredia, haustoria, hyphal lengths and hyphal infection area, which are indicators to assess fungal expansion ability, were strictly reduced in TaClpS1-1/2as silenced wheat compared with that in BSMV: $\gamma$ treated wheat, suggesting that TaClpS1 promotes susceptibility of wheat to Pst.

Phytophthora belongs to hemibiotrophic filamentous pathogens, the disease spot size of which in host tissue visually reflects the degree of its infection and suggests the regulation role of host gene to resistance against Phytophthora. For instance, the lesion diameter of catalase-deficient $N$. benthamiana leaves inoculated with $P$. capsici was greater than control plants, indicating that catalase positively regulated host resistance against $P$. capsici [22]. Overexpression of AtRTP5 in N. 


\section{A}
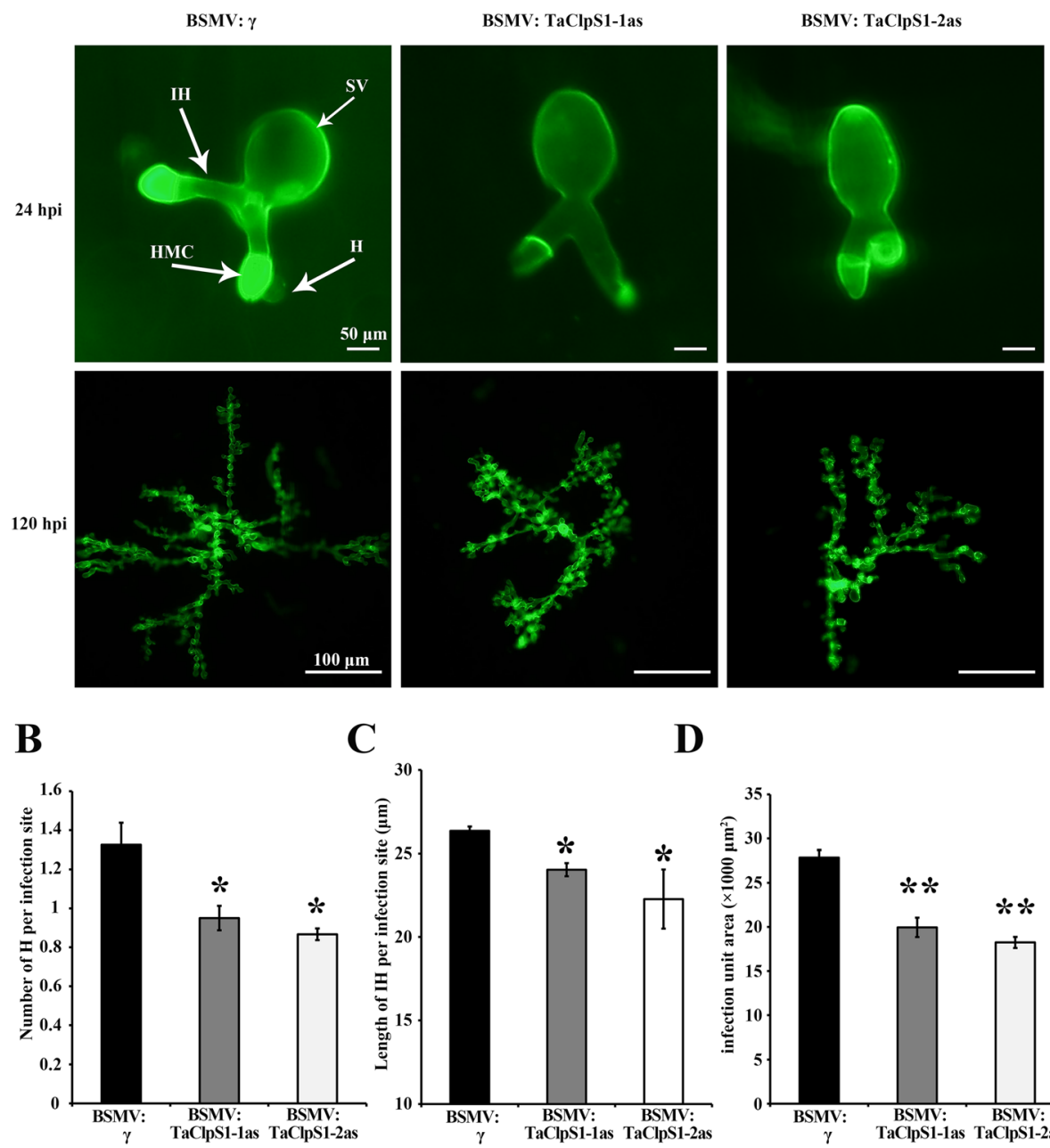

C

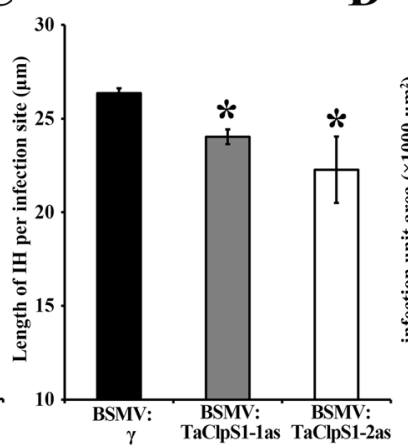

D

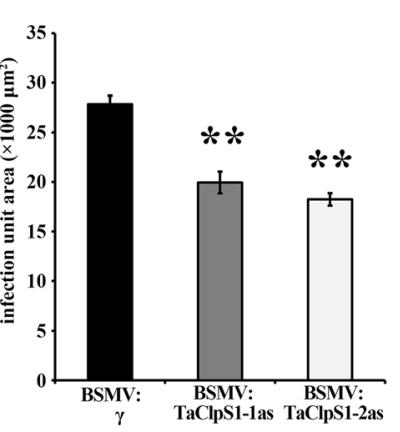

Fig. 6 Silencing of TaClpS1 hinders the expansion of Pst on wheat leaves. a Wheat germ agglutinin (WGA) was used to stain fungal structures in wheat leaves infected with BSMV and Pst. The fungal structures were observed under an autofluorescence microscope. SV, sub-stomatal vesicle; $\mathbb{H}$, infection hypha; $\mathrm{H}$, haustoria; HMC, haustorial mother cell. b The haustoria number per infection site was counted in BSMV-infected wheat leaves inoculated with CYR23 at $24 \mathrm{hpi}$. c The length of IH was measured in BSMV-infected leaves inoculated with CYR23 at 24 hpi. Hyphal length is the distance from the intersection of the sub-stomatal vesicle and the hypha to the tip of the hypha, which was measured by DP-BSW (units in $\mu \mathrm{m})$ program. $\mathbf{d}$ The infection area was measured in BSMV-infected leaves inoculated with CYR23 at 120 hpi using DP-BSW program (units in $\mu \mathrm{m}^{2}$ ). Results shown here were derived from three biological replicates and each replication included 40 individual infection sites. Each data denote the means \pm standard deviation of three biological replicates. Asterisks $(P<0.05)$ or double asterisks $(P<0.01)$ indicate significant differences from BSMV: $\gamma$ treatment at the same time points calculated by unpaired two-tailed Student's $t$-test

benthamiana leaves resulted in the increase of lesion diameters when inoculated with $P$. infestans, indicating that AtRTP5 plays a negative role in regulating plant resistance against Phytophthora [23]. Our results showed that, transient overexpression of TaClpS1 in $N$. benthamiana enhanced infection of $P$. parasitica (Fig. $7 \mathrm{~b})$, suggesting that $\mathrm{TaClpS} 1$ promotes susceptibility of N. benthamiana to $P$. parasitica.

$\mathrm{H}_{2} \mathrm{O}_{2}$ accumulation area and the average necrotic area per infection site in TaClpS1-1/2as silenced wheat were significantly increased, implying that disease resistance was enhanced in TaClpS1 silenced wheat plants. PR proteins are generally considered as marker genes in HRs and are necessary for resistance $[24,25]$. Herein, the transcript levels of TaPR1 and TaPR2 were detected in TaClpS1 silenced wheat plants and negative control infected with the avirulent Pst CYR23. At the 0 hpi, there were no significant differences in the transcript levels of TaPR1 and TaPR2 in TaClpS1 silenced wheat plants and negative control, revealing that the transcript levels of TaPR1 and TaPR2 were not constitutively induced in non-infected TaClpS1 silenced wheat plants. At $24 \mathrm{hpi}$, the transcript levels of TaPR1 and TaPR2 were significantly induced in TaClpS1 silenced wheat and negative 


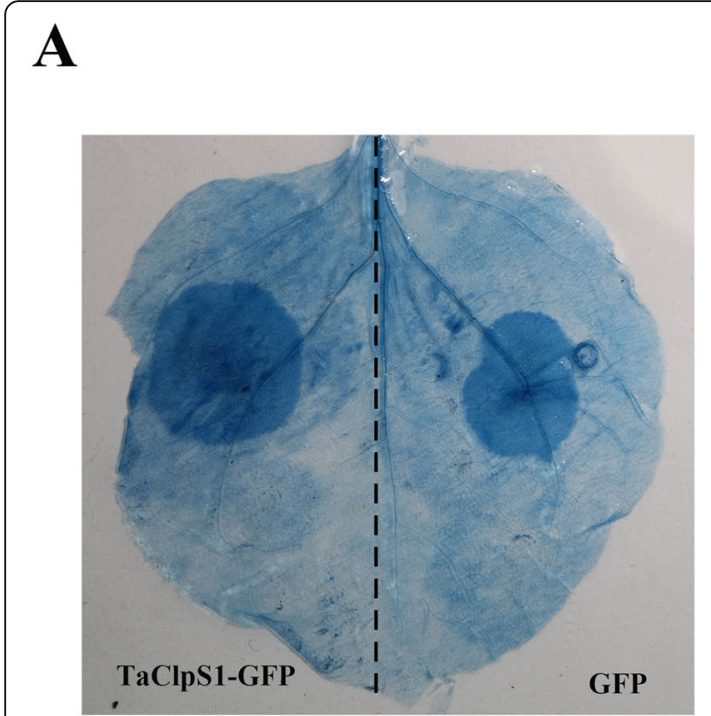

B

C

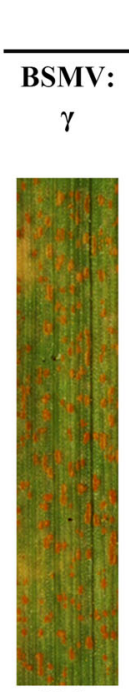

204
CYR31

BSMV: TaClpS1 -1 as

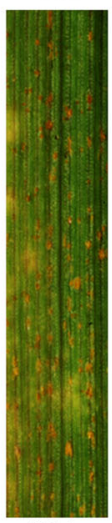

127
BSMV:

TaClpS1

-2as

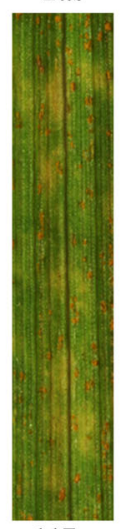

117

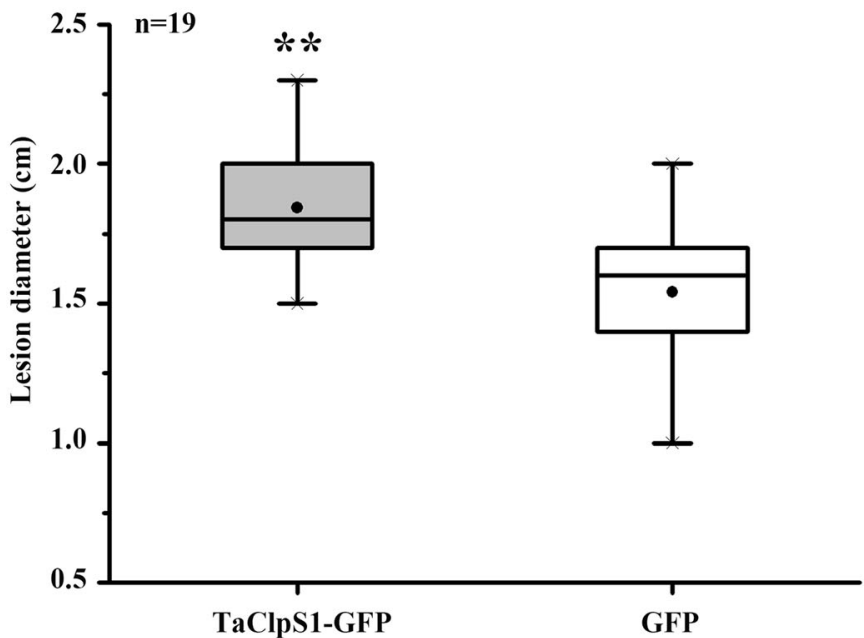

D

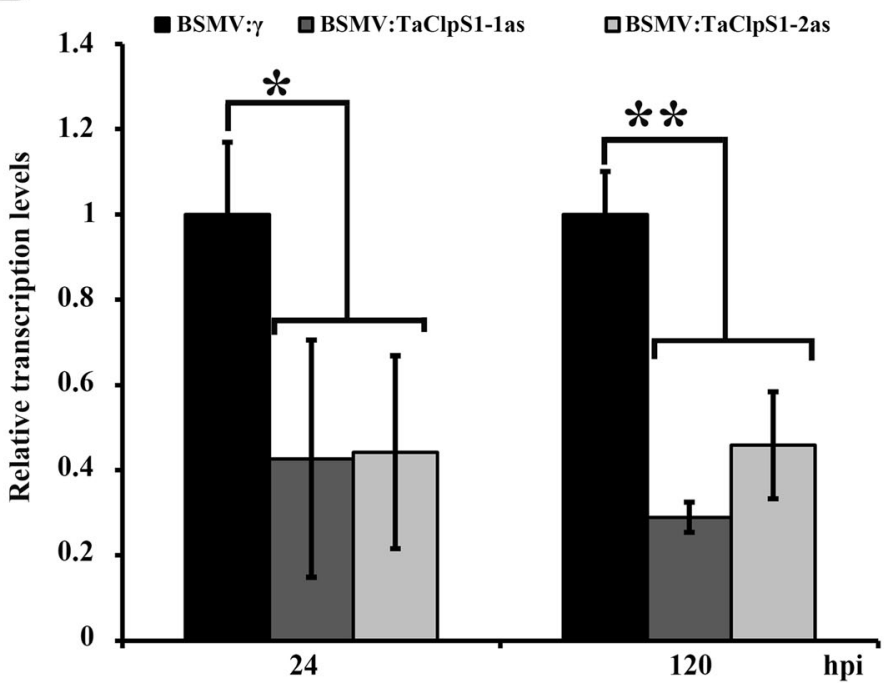

Fig. 7 TaClpS1 facilitates the infection of P. parasitica and Pst in N. benthamiana and wheat, respectively. a The leaves infiltrated with TaClpS1 or GFP were stained with trypan blue to visualize the colonization of $P$. parasitica. $\mathbf{b}$ The lesion diameters of $P$. parasitica infection in the leaves infiltrated with TaClpS1 or GFP were analyzed at $36 \mathrm{hpi}$. Each boxplot illustrates the lesion diameters measured from 19 leaves. The experiment was repeated two times with similar results. The upper quartile, median, and lower quartile are shown in each box, while the bars outside the box indicate the 1st and 99th percentiles. c Phenotypes of the fourth leaves of BSMV-infected wheat plants and further inoculated with Pst isolate CYR31 at $12 \mathrm{dpi}$. Numbers below the leaves indicate the number of uredia. $\mathbf{d}$ Relative transcript levels of TaClpS1 in TaClpS1-silenced plants inoculated with Pst isolate CYR31. The comparative threshold $\left(2^{-\Delta \Delta C T}\right)$ approach was used to measure the relative transcript levels of TaClpS1. Data were normalized against the transcription level of TaEF-1a, and visualized as the fold changes compared to control at 0 hpi. BSMV: $\gamma$ infected wheat leaves were used as control. Each data represent means \pm standard deviation of three independent biological replications. Single asterisks $(P<0.05)$ or double asterisks $(P<0.01)$ denote significant differences from BSMV: $\gamma$ treatment calculated by unpaired two-tailed Student's $t$-test

controls compared to that at $0 \mathrm{hpi}$, while the transcript levels of TaPR1 and TaPR2 were remarkably greater than that in controls. Considering the fact that the accumulation of PR proteins following a pathogen attack is closely associated with the accumulation of salicylic acid (SA) [26], we speculate that the increase of TaPR1 and TaPR2 was accompanied by an increase of SA in TaClpS1 silenced wheat inoculated with Pst CYR23. Additionally, SA, as the representative immune signal, is synthesized in Arabidopsis chloroplasts $[27,28]$, in which $\mathrm{TaClpS1}$ was localized. Taken together, the findings suggest that TaClpS1 
could play a negative role in the SA mediated resistance of wheat to Pst.

Glutamyl-tRNA reductase in Arabidopsis chloroplast has been identified by affinity purification as a candidate substrate of AtClpS1 [17, 29]. Based on the result that glutamyl-tRNA reductase TaHEMA1 in wheat was shown to interact with TaClpS1 using yeast two-hybrid technique, we inferred that TaHEMA1 may function as a substrate of TaClpS1 in wheat. Glutamyl-tRNA reductase is a control point for tetrapyrrole synthesis [30], and increasing research efforts have revealed that tetrapyrrole biosynthesis is involved in the defense response. For example, tetrapyrrole is the main source of singlet-oxygen generation, and singlet oxygen most likely mediates various biological responses, such as host immunity to pathogens [31]. Taken together, it is reasonable that TaClpS1 interrupts tetrapyrrole synthesis to negatively regulate the response of wheat to Pst via selecting glutamyl-tRNA reductase for Clp degration. Future work will be performed to test our hypothesis.

\section{Conclusions}

This study reports for the first time that cloning, localization analysis, and functional characterization of a ClpS1 homolog from wheat of AtClpS1. Expression of TaClpS1 in wheat was induced during Pst infection. Moreover, silencing TaClpS1 led to a decreased susceptibility of wheat to Pst. In addition, heterologous expression of TaClpS1 in $N$. benthamiana enhanced the infection of $P$. parasitica. These results suggest that TaClpS1 negatively regulates the plant resistance to pathogens.

\section{Methods}

\section{Strains, plant materials and growth}

In this study, Pst isolates CYR23 and CYR31 were used to investigate the transcript levels of TaClpS1 and the VIGS assay of TaClpS1 according to the procedure described previously [32]. Fresh Pst urediospores were collected from wheat infected with Pst. P. parasitica strain ZQ-1 used in this study was routinely maintained on $10 \%$ V8 juice medium at $25^{\circ} \mathrm{C}$ in the dark [33].

Wheat (Triticum aestivum L.) variety Suwon11 (AUS22519) originating from Seuseun Agricultural Experiment Station (Sariwon, Korea) was registered in the Australian Winter Cereal Collection, Tamworth, Australia. Suwon11, containing $\mathrm{YrSu}$ resistance gene [34], is resistant to Pst isolate CYR23. Suwon11 seedlings were grown and maintained in a climatic chamber at $16^{\circ} \mathrm{C}$. Tobacco (Nicotiana benthamiana) plants were grown in growth rooms at 21$25^{\circ} \mathrm{C}$ with a 16-h/8-h light/dark cycle. CYR23, CYR31, Suwon11 seeds and $N$. benthamiana seeds were obtained from the Prof. Zhensheng Kang's Lab (Northwest A\&F University, China) [35]. P. parasitica strain ZQ-1 was obtained from Prof. Yongli Qiao (Shanghai Normal University, China) [33].

\section{Plasmid constructs}

The full length of TaClpS1 was cloned into T-simple19 vector to generate TaClpS1-T construct from wheat cultivar Suwon11 cDNA with TaClpS1-specific primers TaClpS1-F/R (Additional file 3: Table S1). To create the constructs for examining the subcellular localization of TaClpS1, full-length TaClpS1 and TaClpS1 $1 \Delta$ were amplified from the above TaClpS1-T construct and inserted into pCAMBIA1302 or pTF486 vector [36] to generate pCAMBIA1302: TaClpS1-GFP, pCAMBIA1302: TaClpS1 $\Delta-$ GFP and pCaMV35S: TaClpS1-GFP, pCaMV35S: TaClpS1 1 GFP respectively. Primer sequences are reported in Additional file 2: Table S1. For VIGS assay, two approximately 150-bp specific silencing fragments were designed based on the combination of Primer5 and NCBI. Barley stripe mosaic virus (BSMV), is a positive-sense RNA virus with a tripartite genome consisting of RNAs $\alpha, \beta$ and $\gamma$. The two designed fragments were cloned with NotI and PacI restriction sites and inserted into original BSMV: $\gamma$ vector to prepare recombinant plasmids BSMV: TaClpS1-1as and BSMV: TaClpS1-2as using specific primers shown in Additional file 3: Table S1 [37]. Above all constructs were obtained from the Prof. Zhensheng Kang's Lab (Northwest A\&F University, China) [35].

\section{Phylogenetic analysis}

For phylogenetic analysis of $\mathrm{TaClpS1}, \mathrm{ClpS1}$ proteins from other plants were obtained using the protein sequence of Arabidopsis AtClpS1 (GenBank accession no. NP_564937.1) to blast NCBI databases. For TaHEMA1, the copies and other related sequences were obtained from the Ensemble Plant database. The phylogenetic tree was constructed using maximum-likelihood method in MEGA5 software. DNAMAN v.7.0 software (LynnonBiosoft, USA) was used to perform multiple sequence alignments and the conserved $\mathrm{ClpS}$ domain was analyzed using Pfam online (http://pfam.xfam.org/).

\section{RNA extraction and analyses of transcript levels}

The second leaves of the two-leaf stage wheat seedlings were inoculated with Pst isolate CYR23. After inoculation, three independent wheat leaves were sampled at 0 , 12, 24, 48, 72, 96, 120 hpi for extracting RNA. Total RNA was extracted with the Quick RNA isolation Kit (Huayueyang Biotechnology, China, Beijing). About $3 \mu \mathrm{g}$ of the total extracted RNA was used for reverse transcription to cDNA with RevertAid First Strand cDNA Synthesis Kit. For RNA extraction and reverse transcription in VIGS assay of TaClpS1, the methods were as described above. LightCycler SYBR Green I Master Mix was used for the qRT-PCR assay, and the transcript 
levels of genes were normalized to the internal control gene TaEF-1 $\alpha$. The primers used in qRT-PCR assay are listed in Additional file 3: Table S1. The statistical significance was evaluated by unpaired two-tailed Student's $t$-test.

\section{Subcellular localization analysis}

To determine the subcellular localization of TaClpS1 in $N$. benthamiana leaves, A. tumefaciens carrying pCAMBIA1302: TaClpS1-GFP, pCAMBIA1302: TaClpS1 $\Delta$-GFP or pCAMBIA1302: GFP vector at a final $\mathrm{OD}_{600}$ of 0.5 was infiltrated into $N$. benthamiana leaves. Vectors pCAMBIA1302: TaClpS1 $\Delta-$ GFP and pCAMBIA1302: GFP were used as negative controls. The infiltrated $N$. benthamiana were maintained in growth rooms at $21-25^{\circ} \mathrm{C}$ with a $16-\mathrm{h} /$ 8-h light/dark cycle. At $48 \mathrm{~h}$ after agroinfiltration, confocal images were obtained with an Olympus IX83 confocal microscope (Japan) using excitation wavelength of $488 \mathrm{~nm}$ and emission wavelength of $520 \mathrm{~nm}$ for GFP, and excitation wavelength of $561 \mathrm{~nm}$ and emission wavelength of $640 \mathrm{~nm}$ for chloroplast autofluorescence, respectively.

For testing the localization of $\mathrm{TaClpS1}$ in wheat cells, Triticum aestivum Suwon11 seedlings were grown in the glasshouse at $25^{\circ} \mathrm{C}$ for $2-3$ weeks. The fusion constructs pCaMV35S: TaClpS1-GFP, pCaMV35S: TaClpS1 $\Delta$-GFP and pCaMV35S: GFP were independently transformed into wheat protoplasts by polyethyleneglycol (PEG)-calcium method as described previously $[38,39]$. The mixtures containing pCaMV35S: TaClpS1-GFP or pCaMV35S: GFP and wheat protoplasts were incubated at $22{ }^{\circ} \mathrm{C}$. Images were obtained with an Olympus IX83 confocal microscope (Japan) at $24 \mathrm{~h}$ after incubation.

\section{BSMV-mediated gene silencing}

Plasmids BSMV: TaClpS1-1as, BSMV: TaClpS1-2as and BSMV: $\gamma$ were linearized followed by transcribing and capping in vitro using the RiboMAX Large-Scale RNA Production System-T7 and the Ribom7G Cap Analog (both by Promega) according to the manufacturer's instructions. Wheat leaves were inoculated with the capped BSMV transcripts and Pst isolate CYR23 or CYR31 according to the procedure described previously [40]. BSMV: $\gamma$ was used as the negative control. The wheat leaves infected with CYR23 were sampled at 0, 24, and 120 hpi for estimating the transcript levels of TaClpS1 and TaPR1/2, $\mathrm{H}_{2} \mathrm{O}_{2}$ detection, measuring necrotic areas and histological observations in VIGS assay of TaClpS1. The symptoms on the wheat leaves were photographed at $12 \mathrm{~d}$ after inoculation with Pst CYR23 and CYR31. These experiments were repeated at least two times.

\section{DAB staining for $\mathrm{H}_{2} \mathrm{O}_{2}$ detection, measuring necroses}

The wheat leaves inoculated with CYR23 in VIGS assay were sampled and stained in $1 \mathrm{mg} / \mathrm{ml} \mathrm{3,3-diaminobenzi-}$ dine (DAB) solution for $6 \mathrm{~h}$ at $16^{\circ} \mathrm{C}$ under light. After staining, the leaves were clarified in the destaining solution (absolute alcohol: acetic acid glacial, 1:1) for about one week. Then, the decolored wheat leaves were further clarified with chloral hydrate for two weeks. Subsequently, $\mathrm{H}_{2} \mathrm{O}_{2}$ accumulation in the transparent leaves was detected with an Olympus BX-51 microscope under bright-field. Alongside detection of $\mathrm{H}_{2} \mathrm{O}_{2}$ accumulation, necrotic areas were measured under UV channel. The results were obtained from 40 infection sites. The samples were collected from three independent leaves. The experiments were repeated three times. The statistical significance was evaluated by unpaired two-tailed Student's $t$-test.

\section{Histological observations of Pst growth}

For histological observations of Pst growth, the wheat leaves inoculated with CYR23 in VIGS experiments were destained at 24 and 120 hpi in absolute alcohol: acetic acid glacial, 1:1 for about one week. Then, clarified wheat samples were stained with wheat germ agglutinin (WGA) conjugated to Alexa-488 (Invitrogen, USA) as described previously [41, 42]. For each biological replicate, 40 infection sites of each sample from three separate leaves were recorded to assess the number of haustoria, hyphal length and infection area. The statistical significance was evaluated by unpaired two-tailed Student's $t$-test.

\section{$P$. parasitica inoculation}

$N$. benthamiana leaves infiltrated with $A$. tumefaciens carrying pCAMBIA1302: TaClpS1-GFP, pCAMBIA1302: GFP were detached at $36 \mathrm{~h}$ after infiltration and challenged with $P$. parasitica by placing mycelial plugs $(5 \mathrm{~mm}$ diam). The inoculated leaves were maintained in a growth room at $25^{\circ} \mathrm{C}$ in darkness. At $36 \mathrm{hpi}$, the inoculated leaves were stained with trypan blue as previously described [43]. Stained leaves were photographed and the diameters of the lesion area were measured.

\section{Yeast two-hybrid assay}

The recombinant BD-TaHEMA1 vector was constructed by cloning TaHEMA1 full length sequence into pGBKT7 with primers TaHEMA1-BD-F/R, and recombinant ADTaClpS1 vector was constructed by cloning TaClpS1 full length sequence into pGADT7 with primers TaClpS1AD-F/R (Additional file 3: Table S1). For interaction assay, BD-TaHEMA1 and AD-TaClpS1 were cotransformed into yeast strain AH109 by the lithium acetate method following Yeast Protocols Handbook (Clontech), and grown on the SD/-Trp-Leu or SD/ -Trp-Leu-His selection medium. Colonies from SD/ -Trp-Leu-His were picked on SD/-Trp-Leu-His-Ade medium containing $\mathrm{X}$ - $\alpha$-gal again for further selection. 


\section{Supplementary Information}

The online version contains supplementary material available at https://doi. org/10.1186/s12870-020-02762-0

Additional file 1: Figure S1. The structure of the two fragments used for silencing TaClpS1.

Additional file 2: Figure S2. Phylogenetic analysis of TaHEMA1

homologs and interaction of TaHEMA1 and TaClpS1.

Additional file 3: Table S1. Primers used in this study.

\section{Abbreviations}

Clp: Caseinolytic peptidase; Pst: Puccinia striiformis f. sp. tritici; VIGS: Virusinduced gene silencing; HR: Hypersensitive response; ROS: Reactive oxygen species; UPS: Ubiquitin-26S proteasome system; E1: Ubiquitin-activating enzymes; E2: Ubiquitin-conjugating enzymes; E3: Ubiquitin ligases; AAA+: ATPase associated with diverse cellular activities; PEG: polyethyleneglycol; qRT-PCR: quantitative real-time PCR; BSMV: barley stripe mosaic virus; hpi: h post-inoculation; PR: pathogenesis-related; WGA: wheat germ agglutinin; DAB: 3,3-diaminobenzidine

\section{Acknowledgments}

We thank Professor Yongli Qiao from Shanghai Normal University, China for providing Phytophthora parasitica.

\section{Authors' contributions}

JG and ZSK designed experiments. QY, MAI, KYC, SXT and YL performed the experiments. QY and JG wrote the manuscript. All authors have read and agreed to the published version of the manuscript.

\section{Funding}

This study was supported by National Transgenic Key Project of the Ministry of Agriculture of China (2020ZX08009-15B), National Natural Science Foundation of China (31972224), National Key R\&D Program of China (2018YFD0200402), Natural Science Basic Research Program of Shaanxi (2020JZ-13) and the 111 Project from the Ministry of Education of China (No. B07049). The funders played no role in the design of the study and collection, analysis, and interpretation of data and in writing the manuscript.

\section{Availability of data and materials}

All data generated in this study are included in the paper and in the supporting information files. Sequence data of this study are available in the Ensemble Plant database (http://plants.ensembl.org/) under accession numbers TraesCS2A02G022700.1 (TaClpS1) and TraesCS1B02G191200.1 (TaHEMA1), and submitted to NCBI GENEBANK database (https://www.ncbi. nlm.nih.gov/genbank) under accession numbers MW233893 (TaClpS1) and MW233894 (TaHEMA1)

\section{Ethics approval and consent to participate}

Not applicable.

\section{Consent for publication}

Not applicable.

\section{Competing interests}

The authors declare no conflict of interest.

Received: 2 May 2020 Accepted: 1 December 2020

Published online: 10 December 2020

References

1. Striebel F, Kress W, Weberban E. Controlled destruction: AAA+ ATPases in protein degradation from bacteria to eukaryotes. Curr Opin Struct Biol. 2009; 19(2):209-17.

2. Sauer RT, Baker TA. AAA+ proteases: ATP-fueled machines of protein destruction. Annu Rev Biochem. 2011;80(1):587-612.

3. Vierstra RD. The ubiquitin/26S proteasome pathway, the complex last chapter in the life of many plant proteins. Trends Plant Sci. 2003;8(3):135-42.

4. Smalle JA, Vierstra RD. The ubiquitin 265 proteasome proteolytic pathway. Annu Rev Plant Biol. 2004;55(1):555-90.
5. Santner A, Estelle M. The ubiquitin-proteasome system regulates plant hormone signaling. Plant J. 2010;61(6):1029-40.

6. Li W, Zhong S, Li G, Li Q, Mao B, Deng Y, et al. Rice RING protein OsBBI1 with E3 ligase activity confers broad-spectrum resistance against Magnaporthe oryzae by modifying the cell wall defense. Cell Res. 2011;21(5):835-48.

7. Zeng L, Qu S, Bordeos A, Yang CW, Baraoidan M, Yan HY, et al. Spotted leaf11, a negative regulator of plant cell death and defense, encodes a Ubox/armadillo repeat protein endowed with E3 ubiquitin ligase activity. Plant Cell. 2004;16(10):2795-808.

8. Fu D, Ghabrial SA, Kachroo A. GmRAR1 and GmSGT1 are required for basal, $R$ gene-mediated and systemic acquired resistance in soybean. Mol PlantMicrobe Interact. 2009;22(1):86-95.

9. Thompson MW, Maurizi MR. Activity and specificity of Escherichia coli ClpAP protease in cleaving model peptide substrates. J Biol Chem. 1994;269(27): 18201-8.

10. Dougan DA, Truscott KN, Zeth K. The bacterial N-end rule pathway: expect the unexpected. Mol Microbiol. 2010;76(3):545-58.

11. Kirstein J, Moliere N, Dougan DA, Turgay K Adapting the machine: adaptor proteins for Hsp100/Clp and AAA+ proteases. Nat Rev Microbiol. 2009;7(8):589-99.

12. Mogk A, Schmidt R, Bukau B. The N-end rule pathway for regulated proteolysis: prokaryotic and eukaryotic strategies. Trends Cell Biol. 2007; 17(4):165-72.

13. Guo F, Esser L, Singh SK, Maurizi MR, Xia D. Crystal structure of the heterodimeric complex of the adaptor, ClpS, with the N-domain of the AAA+ chaperone. ClpA J Biol Chem. 2002;277(48):46753-62.

14. Zeth K, Ravelli RB, Paal K, Cusack S, Bukau B, Dougan DA. Structural analysis of the adaptor protein ClpS in complex with the N-terminal domain of ClpA. Nat Struct Mol Biol. 2002;9(12):906-11.

15. Lupas AN, Koretke KK. Bioinformatic analysis of ClpS, a protein module involved in prokaryotic and eukaryotic protein degradation. J Struct Biol. 2003;141(1):77-83.

16. Dougan DA, Mogk A, Zeth K, Turgay K, Bukau B. AAAt proteins and substrate recognition, it all depends on their partner in crime. FEBS Lett. 2002;529(1):6-10.

17. Nishimura K, Asakura Y, Friso G, Kim J, Oh S, Rutschow H, et al. ClpS1 is a conserved substrate selector for the chloroplast Clp protease system in Arabidopsis. Plant Cell. 2013;25(6):2276-301.

18. Nishimura K, Apitz J, Friso G, Kim J, Ponnala L, Grimm B, et al. Discovery of a unique Clp component, ClpF, in chloroplasts: a proposed binary ClpF-ClpS1 adaptor complex functions in substrate recognition and delivery. Plant Cell. 2015;27(10):2677-91.

19. Apitz J, Nishimura K, Schmied J, Wolf A, Hedtke B, van Wijk KJ, et al. Posttranslational control of ALA synthesis includes GluTR degradation by Clp protease and stabilization by GluTR-binding protein. Plant Physiol. 2016; 170(4):2040-51.

20. Line RF. Stripe rust of wheat and barley in North America: a retrospective historical review. Annu Rev Phytopathol. 2002;40(1):75-118.

21. Cao Z, Jing J, Wang M, Shang $H$, Li Z. Relation analysis of stripe rust resistance gene in wheat important cultivar suwon 11, suwon 92 and hybrid 46. Acta Bot Boreal-Occiden Sin. 2002;23:64-8

22. Zhang M, Li Q, Liu T, Liu L, Shen D, Zhu Y, et al. Two cytoplasmic effectors of Phytophthora sojae regulate plant cell death via interactions with plant catalases. Plant Physiol. 2015:167(1):164-75.

23. Li W, Zhao D, Dong J, et al. AtRTP5 negatively regulates plant resistance to Phytophthora pathogens by modulating the biosynthesis of endogenous jasmonic acid and salicylic acid. Mol Plant Pathol. 2020;21:95-108.

24. Schaffrath U, Freydl E, Dudler R. Evidence for different signaling pathways activated by inducers of acquired resistance in wheat. Mol Plant-Microbe Interact. 1997;10(6):779-83.

25. Van Loon LC, Van Strien EA. The families of pathogenesis-related proteins, their activities, and comparative analysis of PR-1 type proteins. Physiol Mo Plant P. 1999:55(2):85-97.

26. Vlot AC, Dempsey DA, Klessig DF. Salicylic acid, a multifaceted hormone to combat disease. Annu Rev Phytopathol. 2009:47:177-206.

27. Strawn MA, Marr SK, Inoue K, Inada N, Zubieta C, Wildermuth MC. Arabidopsis isochorismate synthase functional in pathogen-induced salicylate biosynthesis exhibits properties consistent with a role in diverse stress responses. J Biol Chem. 2007;282(8):5919-33.

28. Wiermer M, Palma K, Zhang Y, Li X. Should I stay or should I go? Nucleocytoplasmic trafficking in plant innate immunity. Cellular Microbiol. 2007;9(8):1880-90. 
29. Czarnecki O, Hedtke B, Melzer M, Rothbart M, Richter A, Schröter Y, Pfannschmidt T, Grimm B. An Arabidopsis GluTR binding protein mediates spatial separation of 5 -aminolevulinic acid synthesis in chloroplasts. Plant Cell. 2011;23(12):4476-91.

30. Czarnecki O, Grimm B. Post-translational control of tetrapyrrole biosynthesis in plants, algae, and cyanobacteria. J Exp Bot. 2012;63:1675-87.

31. Tanaka R, Tanaka A. Tetrapyrrole biosynthesis in higher plants. Annu Rev Plant Biol. 2007:58(1):321-46.

32. Kang Z, Huang L, Buchenauer H. Ultrastructural changes and localization of lignin and callose in compatible and incompatible interactions between wheat and Puccinia striiformis. J Plant Dis Protect. 2002;109(1):25-37.

33. Zhang P, Jia Y, Shi J, Chen C, Ye W, Wang Y, et al. The WY domain in the Phytophthora effector PSR1 is required for infection and RNA silencing suppression activity. New Phytol. 2019;223(2):839-52.

34. Chen W, Wu L, Liu T, Xu S, Jin S, Peng Y, Wang B. Race dynamics, diversity, and virulence evolution in Puccinia striiformis f. sp. tritici, the causal agent of wheat stripe rust in China from 2003 to 2007. Plant Dis. 2009;93(11):1093-101.

35. Qi T, Guo J, Liu P, He F, Wan C, Islam MA, Tyler BM, Kang Z, Guo J. Stripe rust effector PstGSRE1 disrupts nuclear localization of ROS-promoting transcription factor TaLOL2 to defeat ROS-induced defense in wheat. Mol Plant. 2019;12(12):1624-38.

36. Yu F, Liu X, Alsheikh M, Park S, Rodermel S. Mutations in SUPPRESSOR OF VARIEGATION1, a factor required for normal chloroplast translation, suppress var2-mediated leaf variegation in Arabidopsis. Plant Cell. 2008;20(7):1786-804.

37. Holzberg S, Brosio P, Gross C, Pogue GP. Barley stripe mosaic virus-induced gene silencing in a monocot plant. Plant J. 2002;30(3):315-27.

38. Ito T, Shinozaki K. The MALE STERILITY1 gene of Arabidopsis, encoding a nuclear protein with a PHD-finger motif, is expressed in tapetal cells and is required for pollen maturation. Plant Cell Physiol. 2002;43(11):1285-92.

39. Li C, Lin H, Dubcovsky J. Factorial combinations of protein interactions generate a multiplicity of florigen activation complexes in wheat and barley. Plant J. 2015;84(1):70-82.

40. Fu Y, Duan $X$, Tang C, Li X, Voegele RT, Wang X, et al. TaADF7, an actindepolymerizing factor, contributes to wheat resistance against Puccinia striiformis f. sp. tritici. Plant J. 2014;78(1):16-30.

41. Ayliffe MA, Devilla R, Mago R, White RG, Talbot MJ, Pryor AJ, Leung H. Nonhost resistance of rice to rust pathogens. Mol Plant-Microbe Interact. 2011;24(10):1143-55

42. Cheng $Y$, Wang $X$, Yao J, Voegele RT, Zhang $Y$, Wang $W$, et al. Characterization of protein kinase PSSRPKL, a novel pathogenicity factor in the wheat stripe rust fungus. Environ Microbiol. 2015;17(8):2601-17.

43. Dong $\mathrm{S}$, Zhang Z, Zheng $X$, Wang Y. Mammalian pro-apoptotic bax gene enhances tobacco resistance to pathogens. Plant Cell Rep. 2008;27(9):1559-69.

\section{Publisher's Note}

Springer Nature remains neutral with regard to jurisdictional claims in published maps and institutional affiliations.

Ready to submit your research? Choose BMC and benefit from:

- fast, convenient online submission

- thorough peer review by experienced researchers in your field

- rapid publication on acceptance

- support for research data, including large and complex data types

- gold Open Access which fosters wider collaboration and increased citations

- maximum visibility for your research: over $100 \mathrm{M}$ website views per year

At $\mathrm{BMC}$, research is always in progress.

Learn more biomedcentral.com/submissions 